\title{
Las niñas rezagadas: dirigir las intervenciones de VIH hacia las más vulnerables
}

Judith Bruce

Population Council

Follow this and additional works at: https://knowledgecommons.popcouncil.org/departments_sbsr-pgy

Part of the Demography, Population, and Ecology Commons, Family, Life Course, and Society

Commons, Gender and Sexuality Commons, and the International Public Health Commons

How does access to this work benefit you? Let us know!

\section{Recommended Citation}

Bruce, Judith. 2007. "Las niñas rezagadas: dirigir las intervenciones de VIH hacia las más vulnerables," Promoción de transiciones a la vida adulta sanas, seguras y productivas Resumen no. 23. New York: Population Council. 


\section{Las niñas rezagadas: dirigir las intervenciones de VIH hacia las más vulnerables}

\section{Preparado por Judith Bruce}

A I ingresar en la tercera década de la pandemia de $\mathrm{VIH}$ en el África subsahariana, las mujeres jóvenes - a menudo niñas adolescentes - conforman el nuevo rostro de la enfermedad. En especial, el VIH/SIDA amenaza a aquellas niñas y mujeres jóvenes con limitadas posibilidades sociales y económicas; quienes no pueden evitar, controlar o abandonar las relaciones sexuales inseguras, dentro o fuera del matrimonio.

Las niñas que no asisten a la escuela, en especial las de 10 a 14 años de edad que viven lejos de sus familias, son particularmente vulnerables. Es posible que aquéllas que emigraron recientemente a áreas urbanas, especialmente las trabajadoras domésticas, y las niñas que cuidan de familias infectadas de VIH o actúan como jefas de familia, sufran una importante presión para sostenerse a sí mismas, a sus padres 0 a sus hermanos. Las jóvenes casadas están sometidas a tener relaciones sexuales frecuentes y sin protección, a menudo con parejas mayores, y por lo tanto pueden enfrentarse a un riesgo elevado de infección por VIH.

Las niñas que están en riesgo de ser explotadas sexualmente no constituyen una pequeña minoría; se calculan en cientos de millones. Sin embargo, a pesar de semejante número, las intervenciones existentes no llegan a la mayoría. De hecho, la mayoría de los programas de salud, de desarrollo social, de medios de subsistencia y de juventud no llegan a las niñas más vulnerables que conviven con el $\mathrm{VIH}$.

\section{Determinantes estructurales de riesgo}

La vulnerabilidad de las adolescentes al $\mathrm{VIH}$, en especial en el África subsahariana, tiene su origen en las siguientes condiciones estructurales clave: (1) aislamiento social, (2) ausencia de la escuela durante la adolescencia, (3) matrimonio precoz, (4) actividad sexual insegura, y (5) presión para proporcionar trabajo e ingresos productivos.

\section{Aislamiento social}

Uno de los impedimentos estructurales centrales para muchas niñas y mujeres jóvenes es el alto grado de aislamiento social. En el contexto de la pandemia de VIH, este aislamiento tiene importantes consecuencias. Por ejemplo, existen seis veces más probabilidades de que las niñas más aisladas socialmente en KwaZulu-Natal, Sudáfrica, hayan sido físicamente obligadas a tener relaciones sexuales que las niñas más relacionadas socialmente (Hallman y Diers, 2004). El aislamiento social, la pobreza, la condición de orfandad y la asistencia a la escuela han sido vinculados en forma independiente a la coerción sexual y al intercambio de sexo por regalos, dinero, refugio y alimentos (Hallman 2004 y 2005; Hallman y Diers, 2004). Entre las jóvenes de 18 a 24 años de edad en KwaZulu-Natal, una vida social más activa está correlacionado con una mayor exposición a los mensajes sobre VIH en los medios de comunicación, menos parejas sexuales y mayor probabilidad de haberse hecho un examen de VIH (Hallman y otros, 2007).

De manera constante en todos los grupos de ingresos, las niñas tienen redes de amistades más pequeñas y menos confiables que los varones, y menos espacios seguros y de apoyo donde conocer a sus amigos (Erulkar y otros, 2004b; Hallman y Diers, 2004). En un estudio llevado a cabo recientemente en Kibera, Kenia - una comunidad de 800,000 habitantes y el barrio bajo más grande de África- se halló que de las 90,000 niñas adolescentes de 10 a 19 años de edad que viven allí, sólo aproximadamente una cuarta parte informó tener un sitio seguro para reunirse con otras jóvenes de su misma edad fuera de su casa y de la escuela (Erulkar y Matheka, 2007). En un estudio participativo complementario realizado por niñas líderes se descubrió que menos del $1 \%$ tenía acceso una vez por semana a programas sociales siquiera mínimos en un espacio para niñas solamente. Tan escaso acceso a espacios propios para 
niñas no es sólo una pérdida emocional, sino que también les niega el acceso a potenciales programas que podrían brindarles información, ayudarlas a construir sus habilidades y ofrecerles servicios directamente o por referencia.

\section{Ausencia de la escuela}

Las niñas jóvenes que no asisten a la escuela se enfrentan a amenazas considerables para su salud y seguridad debido a condiciones explotadoras de trabajo en áreas urbanas, y, en las zonas rurales, a la presión para casarse precozmente. Para muchas, escapar de las escasas oportunidades y del matrimonio inseguro hacia perspectivas inciertas en la ciudad constituye un fenómeno relacionaado y peligroso (Erulkar y otros, 2004a y 2004b).

Una revisión global de la matriculación escolar reveló que las niñas solteras actualmente inscritas de 15 a 17 años de edad tenían posibilidades mucho menos que las niñas de la misma edad no inscritas, de tener relaciones sexuales; también tenían índices más bajos de embarazo y infecciones de transmisión sexual (Lloyd 2005).

\section{Matrimonio precoz}

Si continúan los patrones actuales, durante los próximos diez años habrá 100 millones de niñas casadas precozmente. La salud sexual de las niñas casadas está en mayor riesgo que la de las niñas no casadas. Las niñas casadas generalmente tienen parejas de más edad que las parejas de sus contrapartes no casadas sexualmente activas. Deben soportar la intensa presión de quedar embarazadas antes de que sus cuerpos estén preparados para llevar un embarazo, y tienen una frecuencia más alta de actividad sexual que sus pares no casadas sexualmente activas (Clark 2004; Clark, Bruce y Dude, 2006). Las adolescentes casadas tienden a tener redes de pares limitadas o no tener ninguna, movilización social restringida, escasos logros educativos (y prácticamente ninguna opción de educación), y acceso limitado a los medios de comunicación modernos y a los mensajes sobre salud (Erulkar y otros, 2004b).

\section{Condiciones inseguras de actividad sexual}

La exclusión efectiva de las niñas jóvenes de los esfuerzos de prevención de VIH es el fruto de la ignorancia y la negación rotunda de las condiciones de actividad sexual de las niñas en la adolescencia. Una gran parte de dicha actividad tiene lugar dentro del matrimonio, y las niñas casadas suelen describir su iniciación sexual, e incluso sus relaciones sexuales matrimoniales, como forzadas (Erulkar y otros, 2004b). Entre las niñas no casadas, la iniciación sexual también suele ser coercitiva. La mitad de las niñas más pobres de entre 14 y 24 años de edad de las áreas metropolitanas de Durban en KwaZulu-Natal, informaron que su primera relación sexual fue coercitiva (Hallman 2005). Estas niñas no están incluidas en los grupos conven- cionales de alto riesgo, como por ejemplo trabajadores sexuales y consumidores de drogas.

Mayoría de edad sin recursos y en soledad

La adolescencia temprana es una época crítica cuando, para muchas niñas, la vulnerabilidad social y económica representa serias amenazas para su salud. En la mayor parte de los países, la transición de la escuela primaria a la secundaria se produce durante la adolescencia temprana. Muchas familias esperan que las niñas abandonen la escuela y empiecen a trabajar para ayudar a sostener económicamente a la familia. Es posible que las que permanecen en la escuela durante este período deban enfrentar amenazas a su seguridad y respetabilidad, situación que las alienta a abandonar la escuela.

La ausencia de uno o ambos padres -especialmente en la adolescencia temprana - intensifica la vulnerabilidad de las niñas. En muchos países de África subsahariana, la mayoría de las niñas de 10 a 14 años de edad que viven en áreas urbanas viven con sólo uno de sus padres o con ninguno (Chong, Hallman y Brady, 2006). Una condición incluso más extrema puede hallarse entre niñas que no viven con ninguno de sus padres y tampoco asisten a la escuela, como es el caso de más del $8 \%$ de las niñas de ciudad que viven en 18 países subsaharianos, donde se realizan Encuestas demográficas y de salud (EDS) desde el año 2000; esta proporción alcanza el 18\% en Burkina Faso y el $21 \%$ en Mali. Sin recursos, habilidades o protección alguna, estas niñas están expuestas a la explotación tanto laboral como sexual.

\section{Sin alcance a los más vulnerables}

Los adolescentes muy jóvenes (especialmente las niñas) en poblaciones rurales, que no asisten a la escuela y que no viven con sus padres y las niñas casadas son los grupos que están extremadamente abandonadas, o absolutamente ausentes de una gran variedad de programas orientados a la juventud. En una clásica inversión de la distribución de los recursos los adolescentes con mayores ventajas reciben la mayor cantidad de recursos, mientras que quienes tienen menos recursos sociales y se enfrentan a los mayores riesgos reciben la menor cantidad.

En un estudio realizado en forma reciente en Addis Ababa, Etiopía, donde el $30 \%$ de las niñas entre 10 y 14 años vive sin ninguno de sus padres, sólo el $0.5 \%$ de todos los programas llegaba a esas niñas (Mekbib, Erulkar y Belete, 2005). La situación en Etiopía no es inusual. Se observaron patrones similares en Burkina Faso, Guinea Bissau y en Mauritania. En un estudio realizado en Burkina Faso se descubrió que sólo el 19\% de la población atendida por centros de la juventud urbanos estaba formado por niñas (las niñas más jóvenes, de 10 a 14 años de edad, representaban sólo el 3\%), mientras que el $36 \%$ de la "juventud" atendida estaba formado por hombres mayores de 20 años (Lardoux 2006). 
Las niñas casadas, que rara vez asisten a clubes, organizaciones y programas, también tienen acceso limitado a los mensajes de los medios de comunicación, una situación con consecuencias potencialmente graves, ya que la gran mayoría de la actividad sexual de las jóvenes durante la adolescencia —el 94\% en Etiopía - tiene lugar en el contexto del matrimonio (Bruce y Clark, 2003).

Aun cuando los programas lleguen a las niñas casadas, las estrategias actuales de prevención del VIH —abstinencia, disminución de la frecuencia sexual y el número de parejas sexuales, uso de preservativo, conocer el estado de VIH de la pareja y evitar relaciones sexuales con parejas infectadas - son prácticamente inalcanzables para ellas.

\section{Soluciones: avanzar con los recursos existentes}

Existen recursos para abordar esta situación: pueden implementarse buenas leyes; los subsidios pueden extenderse; los programas, y en muchos lugares, una red importante de servicios de salud, sociales y económicos y de iniciativas de la ciudadanía pueden ser reorientados para llegar a estas niñas y construir sus recursos de protección.

\section{Localización de las altas concentraciones de niñas vulnerables}

Debemos esforzarnos más por encontrar e identificar a las niñas y mujeres jóvenes que se concentran en las comunidades con graves epidemias de $\mathrm{VIH}$, de la siguiente manera:

- analizando datos, como los reunidos en las Encuestas demográficas y de salud, para localizar a las niñas de las comunidades rurales y vecindarios urbanos, y complementarlos con evaluaciones cualitativas;

- apoyando las iniciativas de salud infantil y comunitaria que utilizan el contacto puerta a puerta para identificar a las niñas y niños en riesgo, y

- localizando los servicios donde las niñas (y los niños) vulnerables se relacionan o congregan, como por ejemplo las paradas de autobús.

\section{Educación segura hasta la adolescencia}

Es de importancia fundamental lograr que las niñas vayan a la escuela a tiempo y se mantengan allí hasta la adolescencia. Faltar a la escuela, aunque ésta sea de mala calidad, equivale a perder importantes beneficios didácticos, sociales y de salud (y también de salud reproductiva). Una nueva generación de programas universales de educación parece prometedora, aunque muchos sistemas escolares apenas funcionen.

\section{Retrasar y planificar el matrimonio con parejas seguras}

Si se hicieran cumplir las leyes que prohíben el casamiento de niños, las niñas tendrían una mayor posibilidad de retrasar la actividad sexual hasta la edad legal y elegir el momento y las condiciones del matrimonio. Es fundamental reconocer que el matrimonio no es una zona de seguridad sexual (Erulkar y otros, 2004a y 2004b).

Deberían ampliarse los programas piloto que promueven una transición segura y sana al matrimonio. Cuando se les dio la oportunidad, las niñas casadas participaron con entusiasmo en clubes que ofrecen recuperación de la educación, alfabetismo funcional, habilidades para ganarse la vida, capacitación en medios de subsistencia, apoyo efectivo para embarazos y nacimientos seguros, y acceso a servicios de examen y tratamiento de VIH (Erulkar y otros, 2004b).

\section{Espacios sólo para niñas}

Crear espacios dedicados a las niñas constituye una estrategia clave para la transformación de la conciencia de sí mismas y la expansión de su base de conocimientos, y para brindarles una plataforma a partir de la cual ofrecerles servicios vitales de salud, directamente o por referencia.

Los espacios seguros pueden institucionalizarse a un costo relativamente bajo si se establecen en sitios públicos, como por ejemplo centros de jóvenes, centros comunitarios y escuelas, o instituciones voluntarias, entre ellas organizaciones religiosas, iglesias, mezquitas y organizaciones no gubernamentales locales. Todos ellos existen en casi cualquier comunidad, y las inversiones ya han sido hechas, de manera que es posible brindar a las niñas sesiones específicas para su edad y el género.

\section{Creación de un recurso fundamental de mentoras}

Las perspectivas de cambio de las niñas están limitadas donde no existen modelos de conducta o estructuras de tutoría de mujeres mayores para ayudarlas. Las mujeres jóvenes graduadas de la escuela secundaria que no están empleadas constituyen un recurso valioso y poco valorado. La experiencia indica que, incluso en comunidades desfavorecidas, puede reclutarse a mentoras mujeres, generalmente de 17 a 30 años de edad, a cambio de un pequeño estipendio. Estas jóvenes hablan en los idiomas locales, están establecidas y conocen los códigos de la comunidad, y pueden ofrecer una valiosa tutoría a las niñas necesitadas (Binti Pamoja Center 2006; Erulkar y otros, 2006).

Reconsiderar los programas de medios de subsistencia para satisfacer las necesidades de las niñas

Las niñas de todas las comunidades y niveles de ingresos están enormemente interesadas en desarrollar habilidades para ganarse la vida, y expresan interés en la afiliación a grupos, los programas de ahorros y la educación financiera destinados a su edad y contexto. Los lugares de reunión de los programas, creados para niñas y mujeres jóvenes, podrían ser el punto de partida de clubes de ahorros donde éstas podrían adquirir educación financiera, acceso a los ahorros y trabajo digno. Los programas de medios de subsistencia deben estar adaptados a sus 
necesidades; los experimentos de microfinanzas que no prestan suficiente atención a las necesidades sociales de las niñas, y que requieren que se saque un préstamo sin poner énfasis inicial en la educación financiera y en los ahorros, aumentan la vulnerabilidad de las niñas (Population Council 2005; Erulkar y otros, 2006).

\section{Conclusión: recrear las oportunidades de las niñas adolescentes}

Las estructuras de apoyo social y oportunidades de las niñas adolescentes pueden modificarse sistemáticamente, y con ellas, la dimensión de la pandemia. Las niñas son partícipes importantes en este esfuerzo, pero debe incluirse a las familias y a las comunidades. De lo contrario, las mujeres más jóvenes y pobres deberán continuar soportando una parte cada vez mayor y desproporcionada de esta tragedia humana.

\section{Referencias y publicaciones relacionadas}

Binti Pamoja Center. 2006. "Financial literacy education: A curriculum for adolescent girls in Kibera Trainer's Guide." Washington, DC: The Global Financial Education Program.

Bruce, Judith and Shelley Clark. 2003. "Including married adolescents in adolescent reproductive health and HIVIAIDS policy," Background paper presented at WHO/UNFPA/Population Council Technical Consultation on Married Adolescents, Geneva, 9-12 December.

Bruce, Judith and Amy Joyce (eds). Forthcoming. "The girls left behind: The failed reach of current schooling, child health, youthserving, and livelihood programs for girls living in the path of HIV." New York: Population Council.

Chong, Erica, Kelly Hallman, and Martha Brady. 2006. Investing When It Counts: Generating the Evidence Base for Policies and Programs for Very Young Adolescents-Guide and Tool Kit. New York: UNFPA and Population Council.

Clark, Shelley. 2004. "Early marriage and HIV risks in sub-Saharan Africa." Studies in Family Planning 35(3): 149-160.

Clark, Shelley, Judith Bruce, and Annie Dude. 2006. "Protecting girls from HIVIAIDS: The case against child and adolescent marriage." International Family Planning Perspectives 32(2): 79-88.

Erulkar, Annabel S., and James K. Matheka. 2007. Adolescents in the Kibera Slums of Nairobi, Kenya. Nairobi: Population Council.

Erulkar, Annabel, Tekle-Ab Mekbib, Negussie Simie, and Tsehai Gulema. 2004a. The Experience of Adolescence in Rural Amhara Region, Ethiopia. Accra, Ghana: Population Council. 2004b. Adolescent Life in Low Income and Slum Areas of Addis Ababa, Ethiopia. Accra, Ghana: Population Council.

Erulkar, Annabel S., Judith Bruce, et al. 2006. Tap and Reposition Youth (TRY): Providing Social Support Savings, and Microcredit Opportunities for Young Women in Areas with High HIV Prevalence. SEEDS No. 23. New York: Population Council.

Hallman, Kelly. 2004. "Socioeconomic Disadvantage and Unsafe Sexual Behaviors Among Young Women and Men in South Africa." Policy Research Division Working Paper No. 190. New York: Population Council.

___ 2005. "Gendered socioeconomic conditions and HIV risk behaviours among young people in South Africa." African Journal of AIDS Research 41(1): 37-50.

Hallman, Kelly and Judith Diers. 2004. "Social isolation and economic vulnerability: Adolescent HIV and pregnancy risk factors in South Africa." Paper presented at the Annual Meeting of the Population Association of America, Boston, 1-3 April.

Hallman, Kelly, Kasthuri Govender, Emmanuel Mbatha, Rob Pattman, Deevia Bhana, and Jill Walsh. 2007. "Social capital, socioeconomic aspirations, and HIV risk behaviors among poor South African youth." Paper presented at the Third South African AIDS Conference, Durban, 6 June.

Lardoux, Solène. 2006. "Exercice de couverture des activités de paires educatuers au Burkina Faso: Rapport final." New York: UNFPA and Population Council.

Lloyd, Cynthia (ed.). 2005. "Schooling and adolescent reproductive behavior in developing countries." In Growing Up Global:The Changing Transitions to Adulthood in Developing Countries. Washington, DC: The National Academies Press.

Mekbib, Tekle-Ab, Annabel Erulkar, and F. Belete. 2005. "Who are the targets of youth programs?: Results of a capacity building exercise in Ethiopia." Ethiopian Journal of Health Development 19(1): 60-62.

Population Council. 2005. "Building assets for safe, productive lives: A report on a workshop on adolescent girls' livelihoods." Compiled by Erica Chong. New York: Population Council.

___ 2006. "How to conduct a coverage exercise: A rapid assessment tool for programs and services." New York: Population Council.

\section{Donantes}

Dorothy and Lewis Cullman Foundation, UK Department for International Development (DFID), The Dickler Family Foundation, EMpower (The Emerging Markets Foundation), The Ford Foundation, Bill \& Melinda Gates Foundation, Nike Foundation, United Nations Foundation, United Nations Population Fund (UNPFA)

\section{Para obtener más información o copias de los resúmenes de esta serie, escriba a publications@popcouncil.org Para consultar sobre recursos adicionales, visite www.popcouncil.org/pgy}

Population Council

One Dag Hammarskjold Plaza

Nueva York, NY 10017 E.U.A.

(C) 2007 The Population Council, Inc. 